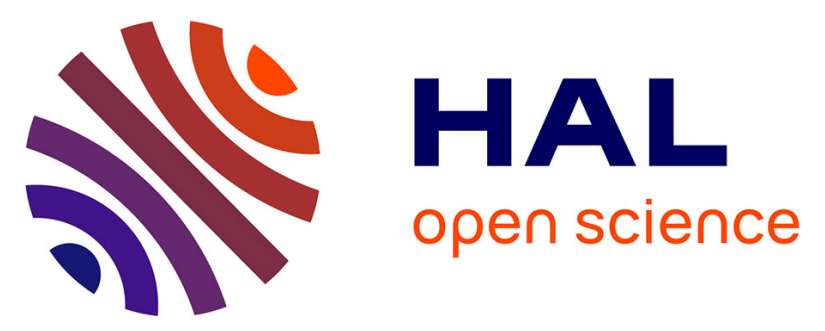

\title{
Field-free molecular alignment of asymmetric top molecules using elliptically polarized laser pulses
}

\author{
A. Rouzee, S. Guerin, O. Faucher, B. Lavorel
}

\section{To cite this version:}

A. Rouzee, S. Guerin, O. Faucher, B. Lavorel. Field-free molecular alignment of asymmetric top molecules using elliptically polarized laser pulses. Physical Review A : Atomic, molecular, and optical physics [1990-2015], 2008, 77, pp.043412. hal-00397189

\section{HAL Id: hal-00397189 \\ https://hal.science/hal-00397189}

Submitted on 19 Jun 2009

HAL is a multi-disciplinary open access archive for the deposit and dissemination of scientific research documents, whether they are published or not. The documents may come from teaching and research institutions in France or abroad, or from public or private research centers.
L'archive ouverte pluridisciplinaire HAL, est destinée au dépôt et à la diffusion de documents scientifiques de niveau recherche, publiés ou non, émanant des établissements d'enseignement et de recherche français ou étrangers, des laboratoires publics ou privés. 


\title{
Field-free molecular alignment of asymmetric top molecules using elliptically polarized laser pulses
}

\author{
A. Rouzée, S. Guérin, O. Faucher, and B. Lavorel \\ Institut Carnot de Bourgogne, UMR 5209 CNRS-Université de Bourgogne, \\ 9 Av. A. Savary, BP 47 870, F-21078 Dijon Cedex, France
}

\begin{abstract}
We show theoretically that a short specific elliptically polarized laser pulse driving an asymmetric top molecule can induce postpulse revivals of three-dimensional (3-D) alignment. By choosing the field ellipticity resulting in the best compromise between the alignment of two molecular axes, we demonstrate that efficient 3-D alignment can be achieved at low temperature. In the experiment, the field-free alignment of moderately cool ethylene molecules is probed by using a technique based on the optical Kerr effect. Control of 3-D field-free alignment opens the door to a large range of applications in chemistry as well as in molecular optics.
\end{abstract}

PACS numbers: $37.10 . \mathrm{Vz}, 42.50 . \mathrm{Hz}, 42.50 . \mathrm{Md}$

\section{INTRODUCTION}

Guiding atoms and molecules by laser fields is the subject of intense research. Controlling the rotational degree of freedom of molecules is one important aspect (see [1] for a review, and $[2,3]$ ). In physics and photochemistry, the effect of interactions with laser fields depends on the relative angle between the molecular axes and the polarization of the laser field. This is particularly prominent for phenomena that rely on intense laser fields like for instance strong field ionization [4], high-order harmonic generation [5], or Coulomb explosion. Aligning or orienting molecules is interesting for study of stereodynamic effects in surface adsorption processes [6]. Many applications can be expected in Chemistry. For example, the yield of a reaction can be enhanced by manipulating the relative orientation of reagents. One reaction channel can also be favoured with respect to the others.

It is well known that adiabatic non-resonant laser pulses (of duration $\tau \gg \hbar / B$, with $B$ the rotational constant of the molecule) align molecules during the interaction with the field, while short pulses $(\tau \ll \hbar / B)$ yield postpulse transient alignment revivals. The latter has been intensively studied both theoretically and experimentally in the last years, principally in linear molecules. However, since most applications in chemistry involve asymmetric top molecules, it is necessary to perform experiments in order to control their rotational motions. With this aim, linearly polarized lasers have been used to align the strongest polarizability axis of asymmetric top molecules right after the pulse in the non-adiabatic regime $[7,8]$. Three-axis adiabatic alignment has also been demonstrated [9] using elliptically polarized long pulses. The drawback of adiabatic alignment is that any envisaged further applications might be spoiled by the strong field of the alignment laser. Fieldfree three dimensional alignment has been studied theoretically [10]. By comparing different strategies, the authors have shown that for ethylene at low temperature $(4 \mathrm{~K})$ the use of two orthogonally polarized, timeseparated laser pulses, is preferable to an elliptically po- larized short pulse. This method has been used recently to observe a field free 3-D alignment of $\mathrm{SO}_{2}$ molecules right after the pulses [11].

In this work, we show that a single elliptically polarized short pulse of appropriate ellipticity allows one to achieve efficient field-free 3-D alignment on a rotational revival. We present a theoretical study in which we find the ellipticity that maximizes simultaneously the alignment of two molecular axes, and therefore $3-\mathrm{D}$ alignment. The effect is calculated for ethylene $\left(\mathrm{C}_{2} \mathrm{H}_{4}\right)$ and compared to a model molecule of optimized polarizability and the same rotational structure. Furthermore, we demonstrate that measurements of the refractive indices modified by the molecular orientation (Langevin contribution) can be used to estimate the 3-D alignment of the molecule, as it has already been shown in the case of one-dimensional alignment [8].

\section{METHOD AND NUMERICAL RESULTS}

When a short non-resonant electric field is applied to a molecule, the interaction between the molecular polarizability and the field produces a rotational wave packet in the ground vibronic state of the molecule. After the extinction of the field, the rotational eigenstates that compose the wave packet periodically rephase and lead to recurring alignment of the molecular axis. This alignment depends on parameters like the rotational constants, the temperature of the gas sample [12], and the pulse shape [14]. Another important parameter is the state of polarization of the electric field. Whereas a linearly polarized electric field can only align one axis of the molecule at a particular time, an elliptically polarized laser pulse offers the possibility to control the alignment of all axes for an asymmetric top molecule.

We consider an elliptically polarized laser field $\overrightarrow{\mathcal{E}}(t)$ propagating in the $\vec{e}_{z}$ direction,

$$
\overrightarrow{\mathcal{E}}(t)=\mathcal{E}(t)\left(a \cos \omega t \vec{e}_{x}+b \sin \omega t \vec{e}_{y}\right),
$$

where $\mathcal{E}(t)$ is the amplitude, $\omega$ the angular frequency, 
and $a$ the parameter representing the half-axis of the ellipse along the laboratory $x$-axis (whereas $b$ corresponds to the $y$-axis) with $a^{2}+b^{2}=1$ and $b>a$. The molecule is assumed to be in its lower vibrational state, in the electronic ground state. For a three-dimensional rigid rotor, with no resonant coupling with excited electronic states nor with vibrational states, the Hamiltonian is given by $[15]$

$$
H=H_{0}-\frac{1}{2} \overrightarrow{\mathcal{E}}(t) \cdot \vec{\alpha} \overrightarrow{\mathcal{E}}(t),
$$

where $H_{0}=B_{Z} J_{Z}^{2}+B_{X} J_{X}^{2}+B_{Y} J_{Y}^{2}$ is the rotational Hamiltonian of the free rotor, $B_{\Gamma}$ the rotational constants, $J_{\Gamma}$ the components of the angular momentum operator of the principal axes of inertia $(\Gamma=X, Y, Z)$, and $\vec{\alpha}$ is the dynamical polarizability tensor which includes the contribution of the excited electronic states. For near infrared excitation the dynamical polarizability can be approximated by the static one. The effective Hamiltonian can be written as

$$
H_{\mathrm{eff}}(t)=H_{0}-\frac{1}{4} \mathcal{E}^{2}(t)\left(\alpha_{x x} a^{2}+\alpha_{y y} b^{2}\right),
$$

with $\alpha_{\gamma \gamma}$ the diagonal laboratory-fixed components of the polarizability $(\gamma=x, y, z)$. We express these components as a function of the diagonal molecule-fixed components using the director cosines $\cos \theta_{\gamma \Gamma}$ defined as $\gamma=\sum_{\Gamma} \cos \theta_{\gamma \Gamma} \Gamma: \alpha_{\gamma \gamma}=\sum_{\Gamma} \cos ^{2} \theta_{\gamma \Gamma} \alpha_{\Gamma \Gamma}$. The properties $\sum_{\Gamma} \cos ^{2} \theta_{\gamma \Gamma}=1$ and $\sum_{\gamma} \cos ^{2} \theta_{\gamma \Gamma}=1$ permit different writings of the interaction potential. Choosing respectively $Z$ and $Y$ the molecular axes of major and minor polarizability leads to the most appropriate writing (up to irrelevant terms that do not multiply a director cosine operator)

$$
\begin{aligned}
V_{\mathrm{int}}= & \frac{1}{4} \mathcal{E}^{2}(t)\left\{\Delta \alpha_{Z X}\left[a^{2} \cos ^{2} \theta_{z Z}-\left(b^{2}-a^{2}\right) \cos ^{2} \theta_{y Z}\right]\right. \\
& \left.-\Delta \alpha_{X Y}\left[a^{2} \cos ^{2} \theta_{z Y}-\left(b^{2}-a^{2}\right) \cos ^{2} \theta_{y Y}\right]\right\}
\end{aligned}
$$

with $\Delta \alpha_{\Gamma \Gamma^{\prime}} \equiv \alpha_{\Gamma \Gamma}-\alpha_{\Gamma^{\prime} \Gamma^{\prime}}$. Equation (4) generalizes in a direct way the interaction potential of a linear molecule, for which we would have $\Delta \alpha_{X Y}=0$ with $Z$ the molecular axis. It has been shown for a linear molecule [16] that the ellipticity satisfying $a^{2}=b^{2}-a^{2}$ (for $b^{2}>a^{2}$ ), i.e. $b^{2}=2 / 3$, gives a dynamically alternating alignment of equal weights along the $y$ - and $z$-axis. For an asymmetric rotor, the first line of (4) shows that $b^{2}=2 / 3$ leads furthermore to the alignment of the most polarizable $Z$-axis that alternates between the $z$-and $y$-axis (if we assume $\left.\left|\Delta \alpha_{Z X}\right| \gg\left|\Delta \alpha_{X Y}\right|\right)$.

Equation (4) shows the additional feature of simultaneous alignment of the $Z$ and $Y$ molecular axes. Using the argument that the molecule will be aligned first at the minimum of the potential, we can infer that the molecular $Z$-axis ( $Y$-axis) will be aligned right after the pulse along the $y$ direction ( $z$ direction) [due to the second and third term in the right hand side of Eq. (4)]. Alignment of these two axes will be strong if the energy is shared (a) 1-D alignment
C-H bonds

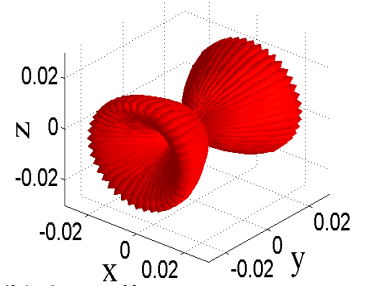

(b) 3-D alignment

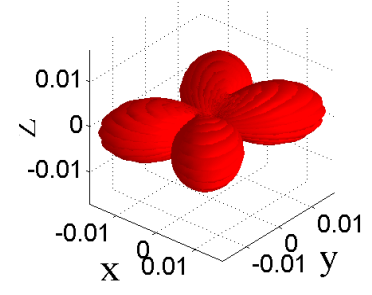

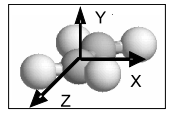

Molecular axes
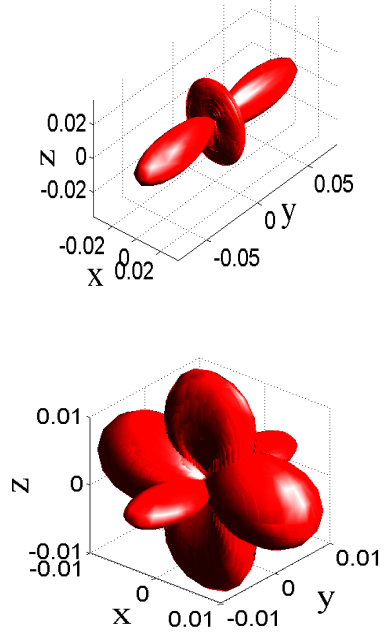

FIG. 1: (Color online) Angular distributions of the C-H bonds (left column) and molecular axes (right column) calculated at $t=8.3 \mathrm{ps}$ after the excitation of an ethylene molecule by a pulse of peak intensity $I=20 \mathrm{TW} / \mathrm{cm}^{2}$ and pulse duration $\tau_{\mathrm{FWHM}}=100 \mathrm{fs}$. The temperature is $T=0.1 \mathrm{~K}$. (a) Linearly polarized laser pulse $\left(a^{2}=0\right)$. (b) Elliptically polarized laser pulse with $a^{2}=0.44$. The structure of $\mathrm{C}_{2} \mathrm{H}_{4}$ and the bodyfixed frame is shown in the inset.

equally between the director cosines $\cos \theta_{y Z}$ and $\cos \theta_{z Y}$ in Eq. (4), i.e. when $\Delta \alpha_{Z X}\left(b^{2}-a^{2}\right)=\Delta \alpha_{X Y} a^{2}$. This is satisfied for

$$
a^{2}=\Delta \alpha_{Z X} /\left(\Delta \alpha_{X Y}+2 \Delta \alpha_{Z X}\right) .
$$

Because the field-free alignment of a near-prolate asymmetric top molecule reaches its maximum at an early time, we will show in the next paragraph that condition (5) leads to a 3-D alignment that is maximized at the first rotational revival.

We start the numerical analysis with ethylene at low temperature. The time-dependent Schrödinger equation using potential (4) is solved numerically with the polarizabilities taken from [17]. The rotational constants are $B_{X}=1.001 \mathrm{~cm}^{-1}, B_{Y}=0.828 \mathrm{~cm}^{-1}$, and $B_{Z}=4.864 \mathrm{~cm}^{-1}[18]$. The $Z$-axis is along the $\mathrm{C}=\mathrm{C}$ bond, while the $\mathrm{X}$-axis is within the molecular plane and perpendicular to the $\mathrm{C}=\mathrm{C}$ bond and the $\mathrm{Y}$-axis is perpendicular to the plane (see the inset in Fig. 1). The laser pulse is treated in the sudden approximation, where the pulse strength is characterized by the two dimensionless parameters $\xi_{Z X}=\frac{\Delta \alpha_{Z X}}{4 \hbar} \int \mathcal{E}^{2}(t) d t$ and $\xi_{X Y}=$ $\frac{\Delta \alpha_{X Y}}{4 \hbar} \int \mathcal{E}^{2}(t) d t$. The calculation during the interaction with the pulse is performed in the rotational eigenbasis of a symmetric top molecule defined by $|J, K, M\rangle$, where $\mathrm{K}$ and $\mathrm{M}$ are the projections of the angular momentum along the molecular Z-axis and the laboratory Z-axis, re- 
spectively. For these rotational states, the transitions induced by the potential (4) are restricted by the selection rules $\Delta J=0, \pm 1, \pm 2, \Delta K=0, \pm 2$, and $\Delta M=0, \pm 2$. Since $\mathrm{K}$ is not conserved during the rotation of an asymmetric top molecule, the field-free evolution of the system is preferably calculated in the diagonal basis $|J, \tau, M\rangle$ [19], where $\tau=K_{Z}-K_{Y}$ is the asymmetric quantum number. $K_{Z}$ and $K_{Y}$ are the projections of the angular momentum along the $Z$ and $Y$ molecular axis defined by continuity in the limit of a symmetric-top [20] and can take the values $K_{Y}=J, J, J-1, J-1, \cdots, 1,1,0$, respectively associated to $K_{Z}=0,1,1, \cdots, J-1, J-1, J, J$ (i.e. the inverse sequence of $K_{Y}$ ). The field free evolution of the state vector reads

$$
\begin{aligned}
\left|\Psi_{J_{0}, \tau_{0}, M_{0}}\right\rangle\left(t>t_{f}\right)= & \sum_{J, \tau, M} C_{J, \tau, M}^{J_{0}, \tau_{0}, M_{0}} \\
& \times e^{-i E_{J, \tau}\left(t-t_{f}\right) / \hbar}|J, \tau, M\rangle,
\end{aligned}
$$

where $E_{J, \tau}$ are the energies associated to the rotational state $|J, \tau, M\rangle$. This last expression is then used to calculate the observables needed for the determination of the alignment.

Figure 1 displays the angular distributions calculated for the first rotational revival produced at $t=8.3 \mathrm{ps}$ after the laser pulse. When no field is applied to the molecule, the distributions are spherical; the probability to find any molecular axis in any direction is isotropic. When the electric field is linearly polarized, we see in Fig. 1 (a) (right column) that the angular distribution of the molecular $Z$-axis peaks in the direction of the electric field, the two other axes remaining delocalized in the $x-z$ plane. In this case, only the most polarizable axis is aligned by the field and the $\mathrm{C}-\mathrm{H}$ bonds are isotropically distributed around the $\mathrm{C}=\mathrm{C}$ bond, giving rise to a diabolo-like shape (left column). When the electric field is elliptically polarized with the value $a^{2}=0.44$ that satisfies Eq. (5), Fig. 1 (b) (right column) shows that the angular distribution of the three $X, Y$, and $Z$ molecular axes peaks in the three orthogonal $x, z$, and $y$ directions, resulting in a 3-D alignment of the molecule. The angular distribution of the $\mathrm{C}-\mathrm{H}$ bonds is clearly localized in the $x-y$ plane and reproduces the geometrical shape of the molecule (left column).

In order to quantify the molecular alignment, we use the thermally averaged director cosines

$$
\begin{aligned}
\left\langle\cos ^{2} \theta_{\gamma \Gamma}\right\rangle(t)= & \frac{1}{Q} \sum_{J_{0}, \tau_{0}} g_{J_{0}, \tau_{0}} e^{-\frac{E_{J_{0}, \tau_{0}}}{k T}} \\
& \times \sum_{\left|M_{0}\right| \leq J_{0}}\left\langle\cos ^{2} \theta_{\gamma \Gamma}\right\rangle_{J_{0}, \tau_{0}, M_{0}}(t),
\end{aligned}
$$

with $Q=\sum_{J_{0}, \tau_{0}}\left(2 J_{0}+1\right) g_{J_{0}, \tau_{0}} e^{-E_{J_{0}, \tau_{0}} /(k T)}, k$ the Boltzmann constant, and $T$ the temperature. $\left\langle\cos ^{2} \theta_{\gamma \Gamma}\right\rangle(t) \equiv$ $\left\langle\psi_{J_{0}, \tau_{0}, M_{0}}\left|\cos ^{2} \theta_{\gamma \Gamma}\right| \psi_{J_{0}, \tau_{0}, M_{0}}\right\rangle(t)$ is the expectation value calculated for the state vector $\left|\psi_{J_{0}, \tau_{0}, M_{0}}\right\rangle(t)$ obtained from the Schrödinger equation with the initial condition $\left|J_{0}, \tau_{0}, M_{0}\right\rangle$. For ethylene, the spin degeneracy in the
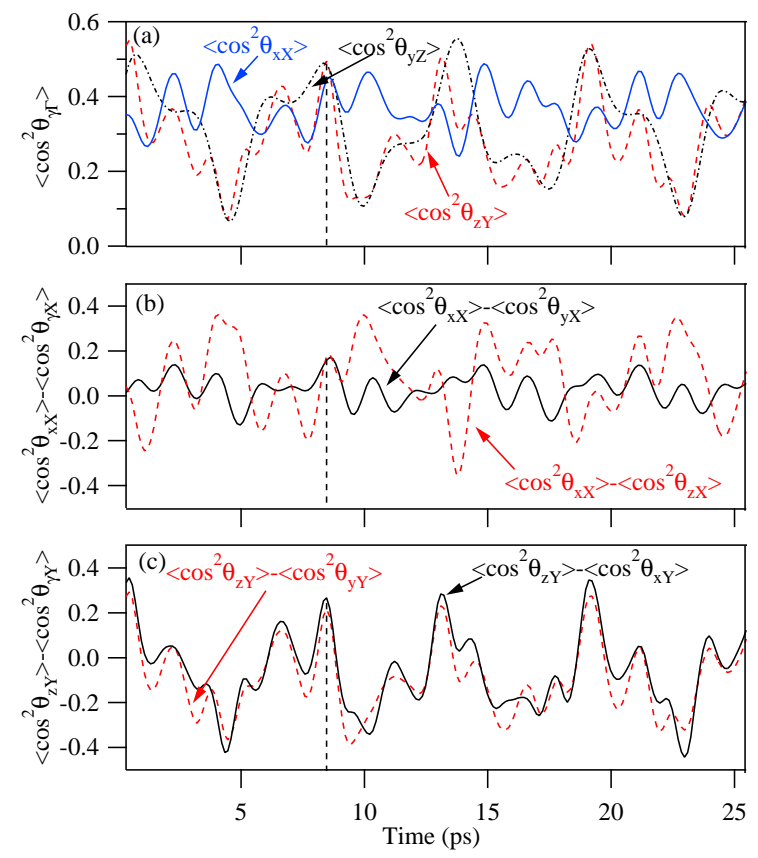

FIG. 2: (Color online) (a) Expectation values of the averaged director cosines of $\mathrm{C}_{2} \mathrm{H}_{4}$ as a function of time for a field ellipticity $a^{2}=0.44$, a peak amplitude $I=20 \mathrm{TW} / \mathrm{cm}^{2}$, a pulse duration $\tau_{\text {FWHM }}=100 \mathrm{fs}$, and a temperature $T=0.1 \mathrm{~K}$. The dashed vertical line indicates the time at which 3-D molecular alignment occurs. (b) and (c) show the difference of the director cosines defined in (9).

ground electronic state results in a specific statistical ensemble of the $J_{0}$ values: if $K_{Z}$ and $K_{Y}$ are both even, the spin degeneracy factor is $g_{J_{0}, \tau_{0}}=7$, otherwise $g_{J_{0}, \tau_{0}}=3$. When $\left\langle\cos ^{2} \theta_{\gamma \Gamma}\right\rangle(t)$ tends to 1 , the $\Gamma$ molecular axis is perfectly aligned in the $\gamma$ direction. On the contrary, when $\left\langle\cos ^{2} \theta_{\gamma \Gamma}\right\rangle(t)$ tends to 0 , the $\Gamma$ molecular axis is totally delocalized in the plane perpendicular to the $\gamma$ axis.

The 3-D alignment is obtained when the three conditions

$$
\left\langle\cos ^{2} \theta_{x X}\right\rangle>1 / 3,\left\langle\cos ^{2} \theta_{y Z}\right\rangle>1 / 3,\left\langle\cos ^{2} \theta_{z Y}\right\rangle>1 / 3
$$

are simultaneously fulfilled. Because $\theta_{x X}, \theta_{y Z}$, and $\theta_{z Y}$ are non independent angles, these conditions are not sufficient to guarantee 3-D alignment. The averaged director cosines must also verify the following criteria [10]

$$
\begin{array}{ll}
\left\langle\cos ^{2} \theta_{x X}\right\rangle-\left\langle\cos ^{2} \theta_{\gamma X}\right\rangle>0, & \gamma=y, z, \\
\left\langle\cos ^{2} \theta_{z Y}\right\rangle-\left\langle\cos ^{2} \theta_{\gamma Y}\right\rangle>0, & \gamma=x, y .
\end{array}
$$

These equations show that the molecular $X$-axis must be preferentially aligned in the $x$ direction rather than in the $y$ and $z$ directions, whereas the $Y$-axis must be preferentially aligned in the $z$ direction. Figure 2 (a) shows the evolution of the three director cosines of Eq. (8) at the temperature $T=0.1 \mathrm{~K}$ (only the state $\left|J_{0}=0, \tau_{0}=0, M_{0}=0\right\rangle$ is populated before the pulse) and for the ellipticity $a^{2}=0.44$. As we can see, the period associated to $\left\langle\cos ^{2} \theta_{y z}\right\rangle$ (describing the alignment of 


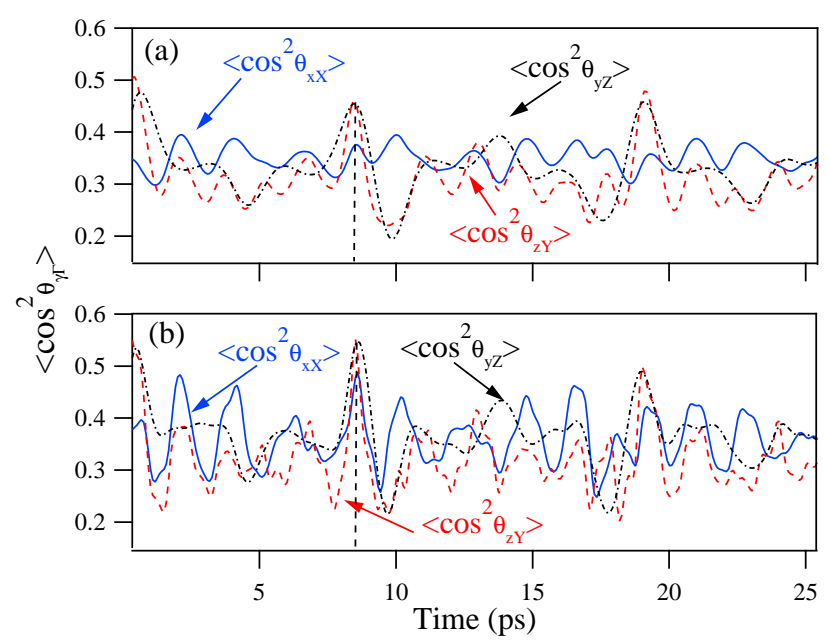

FIG. 3: (Color online) Expectation values of the averaged director cosines as a function of time. Same parameters as in Fig. 2, except $T=4 \mathrm{~K}$. (a) Ethylene $\left(a^{2}=0.44\right)$, (b) model molecule $\left(a^{2}=1 / 3\right.$, see text $)$.

the most polarizable $\mathrm{Z}$-axis along the major y-axis of the ellipse) is close to $\pi \hbar /\left(B_{X}+B_{Y}\right)=9.1 \mathrm{ps}$, the period of the so-called $J$-type transients defined in the high- $J$ limit $[8,21] .\left\langle\cos ^{2} \theta_{x X}\right\rangle$ and $\left\langle\cos ^{2} \theta_{y Y}\right\rangle$ show faster modulations separated by an interval $2 \pi \hbar /\left(4 B_{Z}\right)$, corresponding to a rotation of the molecule about the axis of lower moment of inertia [8], i.e. the $Z$-axis. As in Fig. 1 , we observe on the first $J$-type transient at $t=8.3 \mathrm{ps}$ (see the vertical dashed lines) the synchronous localization of the three molecular axes; all director cosines are in phase and larger than $1 / 3$, satisfying therefore (8). We note in particular that $\left\langle\cos ^{2} \theta_{y z}\right\rangle$ and $\left\langle\cos ^{2} \theta_{z Y}\right\rangle$ have the same amplitude (almost equal to 0.5), due to the specific ellipticity (5) of the field. From the difference of averaged director cosines represented in (b) and (c), we can see that the condition (9) for 3-D alignment is also fulfilled. Conditions (8) and (9) are also satisfied at $t=19.1 \mathrm{ps}$. But for later times, due to the lack of regularity in the rotational-level structure, the alignment of the $X$ molecular axis is smaller. Because the rotational components of the wave packet do not fully rephase after the excitation, the alignment is not periodic. The consequence of the temporal dispersion of the wave packet is the lower efficiency and the rapid decrease of the field-free alignment of an asymmetric top compared to a linear molecule.

We have tested numerically the effects of polarizability and temperature on the $3-\mathrm{D}$ alignment. Figure 3 shows the dynamics of the averaged director cosines calculated at $4 \mathrm{~K}$ for ethylene [Fig. 3 (a)] and for a model molecule of large polarizability asymmetry defined by $\alpha_{X X}=\left(\alpha_{Z Z}+\alpha_{Y Y}\right) / 2$ [Fig. $3(\mathrm{~b})$ ]. The model molecule has been constructed by keeping the rotational constants and the extrema of polarizability $\alpha_{Z Z}$ and $\alpha_{Y Y}$ of ethylene. Compared to $0.1 \mathrm{~K}$ [see Fig. 2(a)], we can see in Fig. 3 (a) that an increase of the rotational tempera- ture results in an expected lower alignment efficiency. In particular, the alignment of the $X$-axis in the $x$ direction is weaker [10]. However, figure $3(\mathrm{~b})$ shows that the alignment efficiency is improved, even though the temperature is higher, when the polarizability tensor is more asymmetric and the field ellipticity $a^{2}=1 / 3$ is chosen as to satisfy condition (5). For this ellipticity, Eq. (4) shows that the energy is equally shared by the two molecular axes and also by both directions $\left(\Delta \alpha_{Z X}=\Delta \alpha_{X Y}\right)$, resulting therefore in a better efficiency for the 3-D alignment.

In this section we have shown that field free 3-D alignment can be produced at a rotational revival using a laser pulse of specific polarization. The alignment occurs mainly at the first J-type transient, during the early time evolution of the system and before significant dephasing of the wave packet that leads to a temporal dispersion of the angular distributions relative to each molecular axis. Compared to others, the first transient is therefore more robust with respect to temperature and intensity. The resulting effect of the elliptic field with respect to $3-\mathrm{D}$ alignment depends strongly on the molecular system, not only on its rotational structure, but more importantly on its polarizability. In particular, the larger the asymmetry of polarizability, the more robust the alignment is with respect to the temperature.

\section{MEASUREMENT OF THE ALIGNMENT}

The non-intrusive technique described in Refs. [22, 23] is used to probe the field-free molecular alignment induced by the elliptically polarized laser pulse. Its principle is based on the modification of the refractive indices resulting from the alignment of the molecules. It can be seen as an orientational contribution to the optical Kerr effect which manifests itself through a retarded response of the medium with respect to the quasi-instantaneous electronic response. In order to measure this effect, we focus a weak time-delayed probe pulse, co-propagating with the alignment pulse (pump), into a gas sample. Due to the alignment dynamics, the medium exhibits recurring transient birefringence that we observe through the depolarization of the probe pulse.

The pump and probe pulses are delivered by a chirped pulse amplified Ti:Sapphire laser operating at $10 \mathrm{~Hz}$. The pulse duration is about $100 \mathrm{fs}$ and the wavelength is centered at $800 \mathrm{~nm}$. The probe laser field propagating along the $\mathrm{z}$-axis is linearly polarized at $45^{\circ}$ with respect to the $y$-axis. In order to change the intensity of the pump, we use a half-wave plate combined with a polarizer. The ellipticity of the pump field can be adjusted by a half-wave plate combined with a quarter-wave plate. Both pulses are focused with a $300 \mathrm{~mm}$ focal lens and cross each other at a small angle $\left(\sim 2^{\circ}\right)$ in a molecular jet of pure $\mathrm{C}_{2} \mathrm{H}_{4}$. The measured beam waists are $40 \mu \mathrm{m}$ and $60 \mu \mathrm{m}$ for the pump and probe, respectively. The depolarization signal of the probe is collected after a polarizer set at $90^{\circ}$ with 
respect to the initial polarization direction.

The signal intensity delivered by the detector in heterodyne detection can be approximated by

$$
I_{\mathrm{sig}}(\tau) \propto 2 \int E_{\mathrm{LO}}(t-\tau) E_{\mathrm{pr}}(t-\tau) \Delta n(t) d t+C,
$$

where $\Delta n(t)=n_{y}(t)-n_{x}(t)$ is the difference of refractive indices experienced by the probe, $E_{\mathrm{LO}}$ is a local oscillator larger than the signal electric field, and $C$ is a term that can be considered as a constant [24]. The alignment signal is obtained by subtracting the constant term measured during the experiment.

With the notation introduced in (1), the birefringence can be written as

$$
\begin{aligned}
\Delta n(t) & =\frac{N}{2 n \mathcal{E}_{0}}\left\{\Delta \alpha_{Z X}\left(\left\langle\cos ^{2} \theta_{y Z}\right\rangle-\left\langle\cos ^{2} \theta_{x Z}\right\rangle\right)\right. \\
& \left.+\Delta \alpha_{Y X}\left(\left\langle\cos ^{2} \theta_{y Y}\right\rangle-\left\langle\cos ^{2} \theta_{x Y}\right\rangle\right)\right\}
\end{aligned}
$$

with $n$ the average value of the refractive index at the probe frequency, $N$ the number density, and $\mathcal{E}_{0}$ the dielectric constant of vacuum. For molecules of large polarizability asymmetry (i.e., $\Delta \alpha_{Z X} \approx-\Delta \alpha_{Y X}$ ), the birefringence experienced by the probe depends on the combined effect of the two alignment of the two molecular $Z$ and $Y$ axes alignment with respect to both field components. In the case of ethylene, where one has $\left|\Delta \alpha_{Z X}\right|>>\left|\Delta \alpha_{Y X}\right|$, the birefringence can be approximated by

$$
\Delta n(t)=\frac{N}{2 n \mathcal{E}_{0}} \Delta \alpha_{Z X}\left(\left\langle\cos ^{2} \theta_{y Z}\right\rangle-\left\langle\cos ^{2} \theta_{x Z}\right\rangle\right) .
$$

For molecules of small polarizability asymmetry, the birefringence signal therefore only provides the information about the angular localization of the $Z$-molecular axis (the $\mathrm{C}=\mathrm{C}$ bond) with respect to the two elliptic field axes. In that respect, the technique does not allow a direct measurement of the 3D alignment of ethylene for which a detection over at least two axes would be necessary. However, since the measured signal is proportional to the difference between the two director cosines $\left\langle\cos ^{2} \theta_{y z}\right\rangle$ and $\left\langle\cos ^{2} \theta_{x z}\right\rangle$, the degree of alignment of the $Z$-axis is intrinsically connected to the field ellipticity responsible for the alignment of the two other axes. The purpose of the next section is to show how the alignment of these two unmeasured axes can be inferred from the measurements of the $\mathrm{C}=\mathrm{C} Z$-axis.

\section{RESULTS AND DISCUSSION}

Figure 4 shows the alignment signal recorded at temperature $T=40 \mathrm{~K}$ with an average intensity $I=$ $80 \mathrm{TW} / \mathrm{cm}^{2}$, for different ellipticity parameters $a^{2}$. When the pulse is linearly polarized with $a^{2}=0$ [Fig. 4 (a)], we observe recurring transients that describe the alignment of the most polarizable axis along the direction of polarization of the pump laser pulse [8]. When the polarization of the pump field is changed, we observe

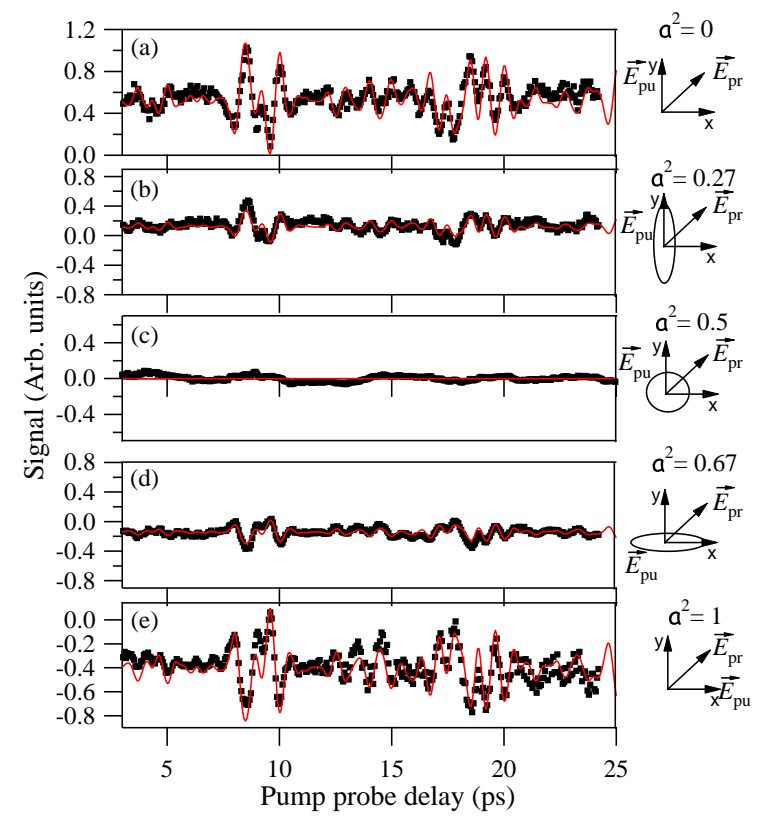

FIG. 4: (Color online) Doted lines: Alignment signals as a function of the pump-probe delay obtained in a molecular jet of $\mathrm{C}_{2} \mathrm{H}_{4}$. The ellipticity of the pump field for different parameters $a^{2}$ (see Sec. II) is depicted on the right-hand side, with $E_{\mathrm{pr}}$ and $E_{\mathrm{pu}}$ denoting the probe and pump pulse, respectively. Full lines: Numerical simulations at $\mathrm{T}=40 \mathrm{~K}$ and $30 \mathrm{TW} / \mathrm{cm}^{2}$ using Eq. (14).

(i) a reduction of the alignment signal from Fig. 4 (a) to Fig. 4 (c), (ii) a minimum for the circular polarization in Fig. 4 (c), (iii) an increase of the signal from Fig. 4 (c) to Fig. 4 (e). Comparing Figs. 4 (a) and (b) and Figs. 4 (d) and (e) we see that the signal is moreover symmetric with respect to $a^{2}=0.5$ [Fig. 4 (c)]. This feature can be explained from Eq. (12). When the field is polarized with $b^{2}>a^{2}$ [Figs. 4 (a) and (b)], we have $\left\langle\cos ^{2} \theta_{y z}\right\rangle>\left\langle\cos ^{2} \theta_{x z}\right\rangle$. The most polarizable axis of the molecule $(Z)$ is preferentially aligned in the $y$ direction. When $b^{2}$ approaches 0.5 [Fig. 4 (c)], the energy distribution over both the $x$ - and the $y$-axis is better balanced, resulting in a decrease of the alignment of the molecular $Z$-axis along the $y$-axis. In the limit of circular polarization, since there is not privileged direction in the $x-y$ plane, we have $\left\langle\cos ^{2} \theta_{y z}\right\rangle=\left\langle\cos ^{2} \theta_{x z}\right\rangle$ and therefore no birefringence. In this case, the alignment of the $Z$-axis occurs along the propagation direction $z$ of the pump field. For $a^{2}>b^{2}$ [Figs. 4 (d) and (e)], the $Z$ axis is preferentially aligned along the $x$ direction with $\left\langle\cos ^{2} \theta_{y z}\right\rangle<\left\langle\cos ^{2} \theta_{x} z\right\rangle$ and the signal is hence the mirror image of the one obtained for $b^{2}>a^{2}$ at the same intensity. These observations can be summarized by the following property $\left\langle\cos ^{2} \theta_{x}\right\rangle(a, b)=\left\langle\cos ^{2} \theta_{y}\right\rangle(b, a)$, with $\Gamma=X, Y, Z$, which gives $\Delta n(a, b)=-\Delta n(b, a)$.

To avoid a prohibitively large use of computer time, we have estimated the averaged director cosines from the 


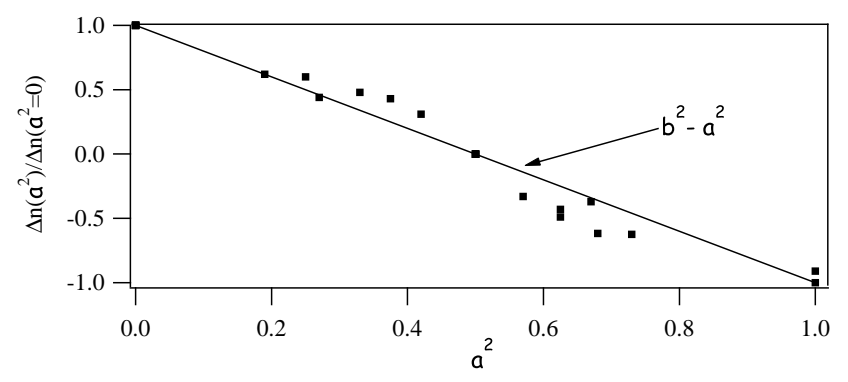

FIG. 5: Amplitude ratio between the signals obtained for an ellipticity $a^{2}$ and for a linear polarization $a^{2}=0$ (squares). Linear regression of equation $b^{2}-a^{2}$ (full line).

following expressions

$$
\begin{aligned}
\left\langle\cos ^{2} \theta_{x \Gamma}\right\rangle-1 / 3 & \approx\left(a^{2}-\frac{b^{2}}{2}\right) f_{\Gamma}(t) \\
\left\langle\cos ^{2} \theta_{y \Gamma}\right\rangle-1 / 3 & \approx\left(b^{2}-\frac{a^{2}}{2}\right) f_{\Gamma}(t) \\
\left\langle\cos ^{2} \theta_{z \Gamma}\right\rangle-1 / 3 & \approx-\frac{1}{2} f_{\Gamma}(t)
\end{aligned}
$$

with $\Gamma=X, Y, Z$ and where $f_{\Gamma}(t)$ is a shape factor computed with a linearly polarized field. These expressions were established for the case of linear molecules in an intermediate field regime, i.e. well below the intrinsic saturation of alignment [25]. We assume that they can be generalized to the three axes of an asymmetric top molecule. The shape factor is independent of the field parameters (such as $a, b$, and the intensity), but not of the other parameters such as the considered molecular axis $\Gamma$ and the temperature for instance. This shape factor features the fact that for moderate intensities, the change of shape of $\left\langle\cos ^{2} \theta_{\gamma \Gamma}\right\rangle-1 / 3$ as a function of the intensity is only due to the change of the permanent alignment. Since here the temperature is rather high leading to a low effective field intensity, we have neglected the permanent alignment effect. Equations (13) show that only the amplitudes of $\left\langle\cos ^{2} \theta_{\gamma \Gamma}\right\rangle$ are modified by the polarization of the electric field and not their temporal behaviors. Using (11) and (13) we can demonstrate that the signal obtained for a given ellipticity $a$ is the one obtained for a linear polarization scaled by the factor $b^{2}-a^{2}=1-2 a^{2}$. One can see from Fig. 5 that this assumption is satisfied experimentally, which confirms that Eqs. (13) are good approximations of the director cosines for the present experimental conditions. In order to extract the averaged director cosines for any ellipticity, we only need therefore to calculate the alignment obtained from a linearly polarized laser pulse and use the expression

$$
\Delta n(t)_{a^{2}}=\left(b^{2}-a^{2}\right) \Delta n(t)_{a^{2}=0},
$$

where $\Delta n(t)_{a^{2}}$ and $\Delta n(t)_{a^{2}=0}$ denote the birefringence produced by the elliptic and linear polarization, respectively. This last equation has been used to simulate the

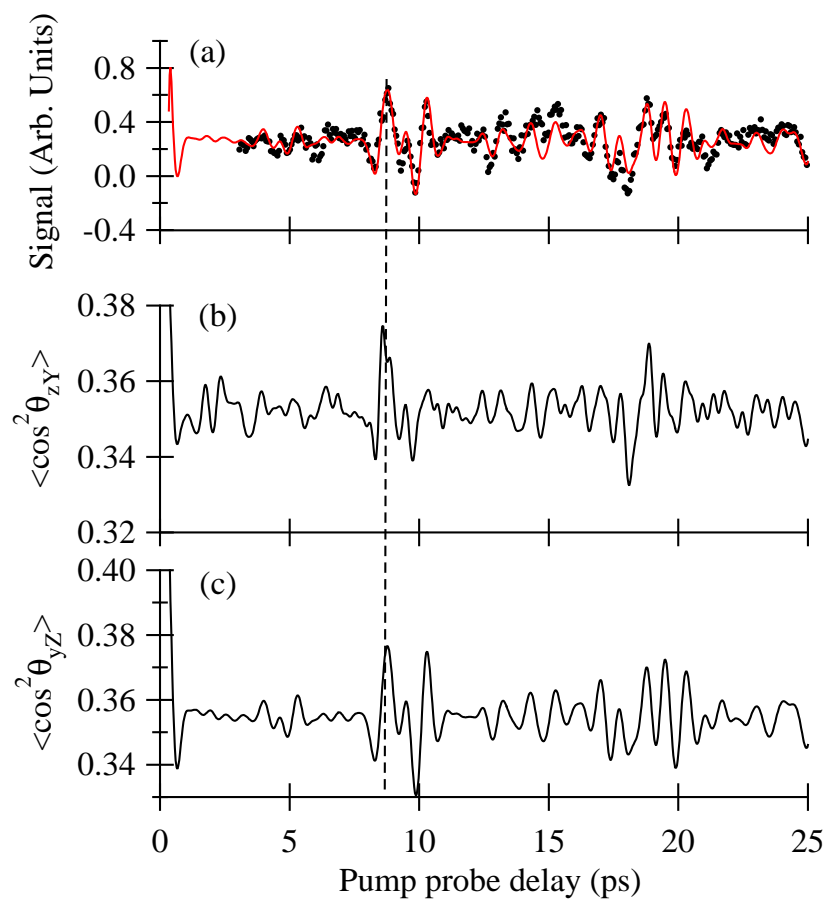

FIG. 6: (Color online) (a) Alignment signal measured in a molecular jet with an ellipticity $a^{2}=0.44$ and an average intensity of $80 \mathrm{TW} / \mathrm{cm}^{2}$ (doted line) and numerical simulation (full lines). Averaged director cosines $\left\langle\cos ^{2} \theta_{y}\right\rangle$ (b) and $\left\langle\cos ^{2} \theta_{z Y}\right\rangle$ (c) calculated from Eqs. (13) for $\mathrm{T}=40 \mathrm{~K}$ and $30 \mathrm{TW} / \mathrm{cm}^{2}$.

results recorded for different ellipticity parameters presented in Fig. 4. In order to get a satisfactory agreement between theory and experiment, the theoretical intensity had to be set at $30 \mathrm{TW} / \mathrm{cm}^{2}$, which is 2.6 times lower than the measured average intensity [26]. As already reported in previous works $[23,27,28]$, this difference can be attributed to the ionization of the molecule that occurs at high intensity. For ethylene, the peak intensity for saturation of ionization is expected to be around 70 $\mathrm{TW} / \mathrm{cm}^{2}[29,30]$, which corresponds to an average intensity of $35 \mathrm{TW} / \mathrm{cm}^{2}$. Considering that the spatial distribution of the laser pulse is not taken into account in the calculation, we have shown that a good approximation consists in comparing the experimental data with the theoretical simulation performed at the average intensity measured in the experiment [27]. Since so far there is no experimental evidence that the ionic species contribute to the alignment signal, we can reasonably assume that the signal observed in Fig. 4 is limited by the saturation of ionization and compare therefore the theoretical intensity $\left(30 \mathrm{TW} / \mathrm{cm}^{2}\right)$ to the average intensity for saturation of ionization $\left(35 \mathrm{TW} / \mathrm{cm}^{2}\right)$.

Figure 6 (a) shows the results obtained at the same laser intensity for the ellipticity parameter $a^{2}=0.44 \mathrm{de}-$ fined according to Eq. (5). As for Fig. 4, the alignment signal has been simulated with a linearly polarized field of a peak intensity $I=30 \mathrm{TW} / \mathrm{cm}^{2}$. Equations (13) 


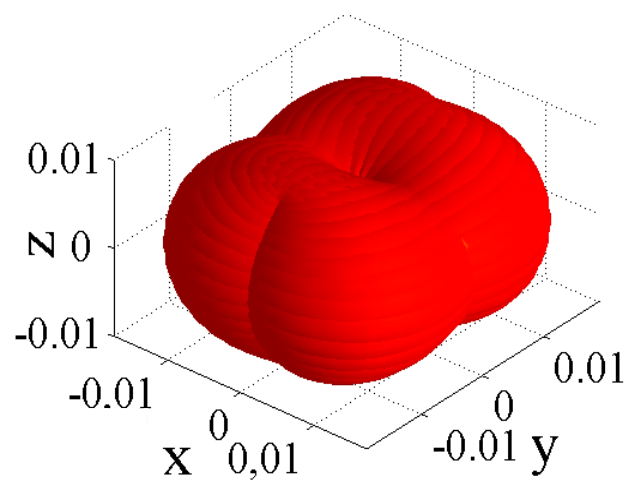

FIG. 7: (Color online) Angular distribution of the $\mathrm{C}-\mathrm{H}$ bonds of ethylene calculated at $t=8.3 \mathrm{ps}$ for an ellipticity $a^{2}=0.44$ and a temperature $T=4 \mathrm{~K}$. The intensity $5 \mathrm{TW} / \mathrm{cm}^{2}$ is chosen as the averaged values of director cosines to be identical to that obtained in the experiment at $T=40 \mathrm{~K}$.

allow us to determine the degree of three-dimensional alignment. Figures 6 (b) and (c) show the averaged director cosines $\left\langle\cos ^{2} \theta_{y z}\right\rangle$ and $\left\langle\cos ^{2} \theta_{z Y}\right\rangle$. The maximum of alignment is observed at $8.7 \mathrm{ps}$ (see the vertical dashed line) with $\left\langle\cos ^{2} \theta_{y Z}\right\rangle=0.37,\left\langle\cos ^{2} \theta_{z Y}\right\rangle=0.37$, and $\left\langle\cos ^{2} \theta_{x X}\right\rangle=0.336$. The relatively weak alignment of the $X$ molecular axis along the $x$-axis is due to the not sufficiently low temperature at which the experiment was conducted. The investigation at temperatures lower than $40 \mathrm{~K}$ was limited in the experiment by the concomitant reduction of the number density in the molecular jet expansion.

Another way to estimate the degree of 3-D alignment achieved in Fig. 6 (a) is to inspect the angular distribution of the $\mathrm{C}-\mathrm{H}$ bonds. Although our theoretical model based on the approximation of Eqs (13) does not allow to simulate this distribution, a qualitative comparison of the numerics and the experiment can be achieved at low temperature in the following manner. The time-dependent Schrödinger equation was solved at $4 \mathrm{~K}$ for a field of ellipticity corresponding to $a^{2}=0.44$. The intensity was adjusted as to result in averaged values of the director cosines identical to those calculated from the experiment conducted at $40 \mathrm{~K}$ (see Figs. 6 (b) and (c) ). The result is presented in figure 7: the angular distribution is a mixture of the diabolo-like shape and the geometrical form of the molecule presented in Fig. 1. The small degree of angular localization observed for the $\mathrm{C}-\mathrm{H}$ bounds corroborates therefore the results of Figs. 4 (b, c) and further emphasizes the temperature issue for 3-D alignment.

\section{CONCLUSION}

We have studied the field-free 3-D alignment of an asymmetric top molecule induced by an elliptically polar- ized short laser pulse. The work is based on a theoretical model that defines the best ellipticity for the molecule to be aligned. The experimental investigation was performed in ethylene, a molecule that is of particular interest in the context of nanoporous solids. The crystal field arising inside the cavity of a nanoporous solid is comparable to the field experienced at the focus of a short laser pulse. Laser induced alignment should therefore provide helpful information about molecules embedded in solids [31]. The molecule was rotationally cooled to $40 \mathrm{~K}$ in a free-jet expansion. The alignment was measured by an optical probe. Although the measurements were conducted at an intensity close to the saturation intensity of ionization, the alignment produced was relatively weak. We show that it is principally due to the combination of the insufficiently low temperature achieved in the molecular jet and the relatively low asymmetry of the molecular polarizability tensor.

The present work stresses the need for strategies to improve postpulse 3-D alignment. A sequence of short pulses has been already used to enhance the degree of alignment of linear molecules [32] and of 3-D molecular alignment [10]. We have numerically investigated the use of an elliptical bipulse, where the second pulse is applied near the maximum efficiency of the first kick, approximately at $t=8.2 \mathrm{ps}$ for ethylene. It has revealed a significant enhancement of the alignment, but at lower temperature than the one investigated experimentally. Phase-shaped laser pulses also offer a solution for enhanced alignment, as it has been shown in a recent theoretical study devoted to the optimization of field-free alignment of linear molecules [13]. More recently, strong 3 -D alignment has been observed combining a long and a short laser pulse [33]. All these promising methods could be adapted in order to produce efficient postpulse molecular 3-D alignment. Finally, the study presented in this paper could be extended to more complex molecules, in particular when the polarizability tensor is not diagonal in the basis of the inertial principal axes [34].

\section{Acknowledgments}

This work was supported by a Marie Curie European Reintegration Grant within the 6th European Community RTD Framework Programme, by the Conseil Régional de Bourgogne, and by the CNRS. 
[1] H. Stapelfeldt and T. Seideman, Rev. Mod. Phys. 75, 543 (2003).

[2] J. Karczmarek, J. Wright, P. Corkum and M. Ivanov, Phys. Rev. Lett. 82, 3420 (1999).

[3] D. M. Villeneuve, S. A. Aseyev, P. Dietrich, M. Spanner, M. Y. Ivanov, and P. B. Corkum, Phys. Rev. Lett. 85, $542(2000)$.

[4] Z. X. Zhao, X. M.Tong, and C. D. Lin, Phys. Rev. A 67, 43404 (2003).

[5] J. Itatani, D. Zeidler, J. Levesque, M. Spanner, D. M. Villeneuve, and P. B. Corkum, Phys. Rev. Lett. 94, 123902 (2005).

[6] L. Vattuone, A. Gerbi, M. Rocca, U. Valbusa, F. Pirani, D. Cappelletti, F. Vecchiocattivi, Angew. Chem. Int. Ed. 43, 5200 (2004).

[7] E Péronne, M.D. Poulsen, C.Z. Bisgaard, H. Stapelfeldt and T. Seideman, Phys. Rev. Lett. 91, 043003 (2003); E Péronne, M.D. Poulsen, H. Stapelfeldt, C.Z. Bisgaard, E Hamilton and T. Seideman, Phys. Rev. A 70, 063410 (2004).

[8] A. Rouzée, S. Guérin, V. Boudon, B. Lavorel and O. Faucher, Phys. Rev. A 73, 033418 (2006).

[9] J. J. Larsen, K. Hald, N. Bjerre, H. Stapelfeldt and T. Seideman, Phys. Rev. Lett. 85, 2470 (2000).

[10] J.G. Underwood, B.J. Sussman, and A. Stolow, Phys. Rev. Lett. 94, 143002 (2005).

[11] K.F. Lee, D. M. Villeneuve, P. B. Corkum, A. Stolow and J. G. Underwood, Phys. Rev. Lett., 97, 173001, 2006.

[12] M. Machholm, J. Chem. Phys., 115, 10724, (2001).

[13] E. Hertz, A. Rouzée, S. Guérin, B. Lavorel, and O. Faucher, Phys. Rev. A 75, 031403(R) (2007).

[14] E. Hertz, A. Rouzée, S. Guérin, B. Lavorel and O. Faucher, Phys. Rev. A, 75, 031403(R), (2007).

[15] B. Friedrich and D. Herschbach, Phys. Rev. Lett. 74, 4623 (1995).

[16] D. Daems, S. Guérin, E. Hertz, H. R. Jauslin, B. Lavorel, and O. Faucher, Phys. Rev. Lett. 95, 063005 (2005).

[17] A. Rouzée, V. Boudon, B. Lavorel, O. Faucher, W. Raballand, J. Chem. Phys. 123, 154309, (2005). By fixing $\alpha_{Z Z}$ to $5.022 \AA^{3}$, we obtain the following polarizabilities: $\alpha_{X X}=3.63 \AA^{3}, \alpha_{Y Y}=3.25 \AA^{3}$.

[18] T. L.Tan, S. Y. Lau, P. P. Ong, K. L. Goh, and H. H.
Teo, J. Mol. Spectrosc. 203, 310 (2000).

[19] M. D. Poulsen, E. Peronne, H. Stapelfeldt, C. Z. Bisgaard, S. S. Viftrup, E. Hamilton, and T. Seideman, J. Chem. Phys. 121, 783 (2004).

[20] Papoušek, D. and Aliev, M. R., Studies in Physical and Theoretical Chemistry, Elsevier, Amsterdam - Oxford New York, (1982).

[21] P. M. Felker and A. H. Zewail, in Femtosecond Chemistry, edited by J. Manz and L. Woste VCH, Weinheim, New York, 1995, Vol. 1, p. 193.

[22] V. Renard, M. Renard, S. Guérin, Y. T. Pashayan, B. Lavorel, O. Faucher, and H. R. Jauslin Phys. Rev. Lett. 90, 153601 (2003).

[23] V. Renard, M. Renard, A. Rouzée, S. Guérin, H. R. Jauslin, B. Lavorel, and O. Faucher, Phys. Rev. A 70, 033420 (2004).

[24] B. Lavorel, O. Faucher, M. Morgen, and R. Chaux., J. Raman Spectrosc. 31, 77 (2000).

[25] A. Rouzée, V. Renard, S. Guérin, O. Faucher, and B. Lavorel, Phys. Rev. A. 75, 013419 (2007).

[26] We define the average intensity as the ratio of the pulse energy to the product of the pulse duration by the beam waist (top-hat approximation).

[27] A. Rouzée, V. Renard, B. Lavorel, and O.Faucher, J. Phys. B 38, 2329 (2005).

[28] V. Loriot, P. Tzallas, E. P. Benis, E. Hertz, B. Lavorel, D. Charalambidis, and O. Faucher, J. Phys. B 40, 2503 (2007).

[29] J. Muth-Bohm, A. Becker, S. L. Chin and F. H. M. Faisal, Chem. Phys. Lett., 337, 313, (2001).

[30] Gao Lirong, Ji Na, Xong Yijia, Tang Xiaoping and Kong Fana'ao, Chinese Science Bulletin, 48, 1713, (2003).

[31] T. Kiljunen, B. Schmidt, and N. Schwentner, Phys. Rev. Lett. 94, 123003 (2005).

[32] M. Leibscher, I. Sh. Averbukh, and H. Rabitz, Phys. Rev. Lett. 90, 213001 (2003).

[33] S. S. Viftrup, V. Kumarappan, S. Trippel, H. Stapelfeldt, E. Hamilton, and T. Seideman, Phys. Rev. Lett. 99, 143602 (2007).

[34] Mikael D Poulsen, PhD. thesis, University of Aarhus, Denmark, 2005. 